\title{
User-Centred Design and Development of an Intelligent Light Switch for Sensor Systems
}

\author{
Boštjan SENIČAR, Helena GABRIJELČIČ TOMC
}

\begin{abstract}
The aim of the research was the user-centred design of an intelligent light switch with a focus on the definition of the natural and intuitive gestures for its manipulation, and to develop a multi-touch user interface and smart touch-based light switch that could be integrated into existing home environments and electrical wiring, with or without an existing intelligent system. After usability testing a prototype of the switch was constructed. The touch-panel, as the main interface, gave the users the ability to control one or more individual lights or light groups. The intuitive touch gestures for controlling the lighting were acquired with paper prototypes that were used for testing, and then integrated in the physical prototype. The results show that user-centred design is a valuable method for creating an intelligent touch-based light switch with a good user experience design, with or without a multi-touch user interface, and thus an approach that should be implemented in the development of a smart product.
\end{abstract}

Keywords: intelligent systems; interaction; multi-touch user interface; smart light switch; user-centred design

\section{INTRODUCTION}

A so-called intelligent house is an intelligent building adapted for everyday housing needs. In addition to the basic operating subsystems that are part of almost every intelligent building system (heating, ventilation, air conditioning, lighting), the design of such houses pays particular attention to luxury, comfort and convenience in terms of housework, multimedia devices, and user-friendly interfaces [1]. Intelligent houses are also called "smart houses", and as with any "smart device", an intelligent house has its own associated subsystems that are simultaneously connected to a larger network (an intranet or the Internet). These subsystems are controlled and managed by the user directly or by remote control via a smartphone, tablet, computer or other device. In addition, an intelligent house should be able to detect various environmental parameters and consequently respond to them in accordance with the proposed algorithms [2, 3]. The whole intelligent house, or only some of its subsystems, can be connected to a global network, the Internet, and in this context the latter is usually referred to as the Internet of Things (IoT) [4, 5]. There are several standards and protocols that have been developed to successfully connect the devices that make up an intelligent system through various types of physical networks. These protocols include $X 10$ [6], UPB (universal power linebus) [7], KNX [8], LonTalk (LonWorks) [9], INSTEON [10], ZigBee [11] and Z-Wave [12].

\subsection{Intelligent Lighting}

Smart lighting, a technology designed for energy efficiency, is one of the most important components of an intelligent house, and significant reductions in energy consumption can be obtained with the appropriate sensor support and automation of lighting. In addition, with the careful set-up and optimised control of lights, the atmosphere of a space can be completely altered or slightly adjusted [13]. However, the user interfaces for lighting control are one of the most vulnerable elements of user interaction design, particularly in intelligent buildings, where it is generally necessary to manage a larger number of functions supporting lighting. Smart light switches usually allow additional control of the lighting, like dimming, while more advanced functions, like timers, group management, and configuration, are still only available through the use of a smartphone app or other smart home management applications.

Some commercial solutions and engineering developments demonstrate the constant improvements that are being made to such systems. Philips Hue, a system of bulbs that is used with a network switch and mobile application, was one of the first breakthrough commercial intelligent lighting systems for home use. The system operates on the ZigBee protocol with a LightLink profile, which allows users to connect devices via a WiFi network. Lighting can then be managed with a mobile application via smartphone or tablet PC $[14,15] . L I F X$ is similar to the Philips Hue system, with the difference that it does not need a separate network switch for connecting, as each bulb is also a switch. The system selects the most appropriate bulb, which is then connected to a local WiFi network and becomes a gateway for communication among the bulbs, the interface and other devices and services [16]. The Ilumi system uses a Bluetooth protocol for connections. Bluetooth has a smaller signal range and is more sensitive to obstacles than $\mathrm{WiFi}$, and thus uses a grid networking topology. Although the system does not include a specific switch, direct and easy connection with the bulb can be obtained with the help of a smartphone or other Bluetooth device. Users can also combine bulbs in groups through the interactive interface [17]. INSTEON provides a comprehensive solution for intelligent house systems. It has its own protocol, a wide range of modules, devices, and routers, and an interface for device control. Plum lightpad switch has a built-in sensor for energy consumption, movement and light, which allows for examinations and comparisons of the energy consumption of individual devices, and automatic lighting activation when the movement of an object in the dark is detected [18].

Rath [19] presented a light control system useful for exterior and interior solutions on an embedded platform (a main board with a microcontroller chip and memory and communication port) with photosensitive detectors (LDR). These enable the adjustment of the lighting system according to light intensity. Barghi et al. [20] proposed a laboratory prototype of an intelligent lighting control system with LEDs for smart homes. The authors 
introduced a manual (PWM signal) and automatic (PID algorithm) control method. The ambient light is measured with photocells and the PWM signal is generated via user control. Mowad et al. [21] presented an Android application and microcontroller for a smart homeautomated control system. In the proposed solution, the control of light is enabled automatically via smartphone or PIR sensor. This subsystem can be installed beside the electrical switches on the wall. The switch connection is controlled by the circuit in the main control board that can also be a multiple circuit. Android version 2.2 is used in the subsystem, with the low API level 8 . The user interface was designed with the ability to directly connect (via Bluetooth) to the main control board (BlueBee) or to a PC/laptop (PROZ). Wen-Tsai et al. [22] demonstrated that a smart LED lighting system in a digital home network can be designed and implemented with a self-adaptive weighted data fusion algorithm. In their system the data communication is performed using the ZigBee standard. A touchscreen computer interface and RS 232/485 server are used to connect the various components, such as a multimeter, wireless light dimmer, IR learning remote module, and so on. Signal processing is performed by a self-adaptive weighted data fusion algorithm. In this system the self-learning mode and remote control are enabled by a single handheld device and WiFi transmission. This system also considers energy efficiency. Moreover, interiors are not the only focus of researchers, as intelligent lighting systems have also been developed for exteriors, such as streets and parks in urban areas [23, 24].

\subsection{User-Centred Design and Interactive User Interfaces}

User-centred design (UCD) is a multidisciplinary process and design methodology wherein the users' (target group's) needs, wants and limitations while interacting with interactive device (interface, solution) are observed, analysed and considered at all stages within the development and lifecycle of the product, with the aim of addressing the whole user experience [25]. UCD in interactive media also involves interaction design, which is not only about the properties and design of the graphic interactive interface, but also about people's actual behaviour when interacting with the interface according to some basic principles of interaction design, such as consistency, visibility, learnability, predictability and feedback [26, 27]. UCD demands that during the development of an interactive user interface the designers take into account the findings of cognitive science [28], and thus draw on an understanding of the fundamental mechanisms and mental processes of perception, memory, attention, learning and reasoning. Usability is a qualitative characteristic of the product which is related to methods for improving the user interface in the design process, and is defined by the following five qualitative components: learnability, efficiency, memorability, errors and satisfaction [27].

A review of the related references revealed that intelligent lighting mostly includes highly defined sensors, protocols and other technological aspects that enable the lighting system environment to be effectively and intelligently implemented in houses, and that user interfaces for lighting control remain vulnerable parts of such systems that still need special attention. Consequently, key issues in related research are the determination of certain essential functions that the light switch should offer to users, and the definition of the types of gestures for manipulation of intelligent lights that should be natural and intuitive to achieve a good user experience. The aim of the present research was thus to implement the UCD methodology in this context, i.e. an iterative design process with a focus on the users' intuitive interactions when performing tasks with a light switch both with and without a graphical user interface. Another aim was to use rapid prototyping in the development of an intelligent light switch. In the experiment the focus was on the acquisition of all the relevant gestures (during predefined tasks) and a definition of the most effective and natural gestures for successful and useful interactions with the switch's interface. Furthermore, based on the resulting user-centred definition of the set of gestures, the goal of this work was to develop a multi-touch user interface and a smart touchbased light switch that could be integrated into existing home environments and electrical wiring (with or without an existing intelligent system). The process included usability testing of the paper prototypes that enabled the planning of the user-experience without conditioning users with any predefined gestures, the definition of the most intuitive gestures for the interactions with the switch, and the construction of a physical switch prototype, which allowed the users to extend its capabilities and functionalities by connecting it with other available smart devices and sensors. The success of the UCD and the usability of the final prototype were further tested with various performance metrics on a small number of participants to confirm the effectiveness of the applied methodology and the suitability of the light-switch prototype.

\section{METHODS}

The procedure used in the experiment is presented in Fig. 1. UCD and development were performed in seven phases: 1. paper prototyping (two paper prototypes were used); 2. gesture definition (with spatial mapping of lights on the user interface and the definition of natural and intuitive gestures); 3. development of the switch (concept design and implementation of the switch functions); 4. testing of the switch's elements (testing of the components, testing on the didactic panel); 5. testing of the switch (with and without the user interface); 6. user-interface design of a graphic interface; and 7. usability testing.

For the purpose of the research work, we decided to limit the number of elements that we wanted to test and focus on user-centred testing and the definition of the most appropriate and intuitive gestures for managing the light switch. The preliminary (primary) interface that was tested and analysed had a touch-panel surface without a predefined graphical screen design and no predetermined zones for interactions. With this procedure it was possible to obtain results that were not conditioned by the user interface design context and pre-defined interactivity.

Therefore, in the first phase a test with the paper prototype was performed and the results of performance metrics [27] were evaluated. In total 20 subjects (10 women and 10 men) were tested: 16 persons aged between 
20 and 30 years, two people aged between 50 and 60 and two persons over 70. A questionnaire was conducted with each user separately to avoid any mutual influences. The entire interview for each user was video recorded (anonymously, showing only the hands of the user) for use in the subsequent analysis. The average duration of the test was 10 minutes. Two paper prototypes were prepared with similar interfaces. The only difference between the prototypes was the presentation of lights that had to be controlled by the users through a "blind" user interface. In the first scenario, where six lights were arranged in a $2 \times 3$ grid, we wanted to analyse the most intuitive reaction and execution of gestures with minimal cognitive demand on the users. In the second scenario, the six lights were arranged in the plane of the room map, as presented in Tab. 1. The users were asked to execute seven different tasks. Tasks A to D were basic, while E, F and G were advanced. The tasks were prepared in order to determine the spatial mapping of lights on the interface and the gestures of the most used commands, such as switching on and off the lights and switching on and off the primary lighting. The purpose of the advanced tasks, such as setting the primary lighting and the automatic switching off the lights with a gradual fade-out, was to examine how the users would activate and combine more operations and how these would be confirmed. Different users performed the sequence of the tasks in Latin square order. After the acquisition of data on the users' performance the video recordings were studied and analysed, and the most commonly suggested gestures for a defined task were identified and are presented in Tabs. 2 to 4 .

\subsection{Concept Design and Development of the Switch}

After testing on the paper prototypes, which enabled the determination of the most intuitive gestures and interactions, the next step in the UCD of the switch was the definition of basic features and capabilities. The results of this dictated the elements that were installed in the switch. The fundamental features were determined on the basis of the actual supply of smart switches and their properties. During the planning and development of the product the principles of effective and good design (ease of use, aesthetics and functionality) were also considered.

Basic functions of the switch: 1. activation/deactivation of an individual light or group of lights; 2. change in light intensity (dimmer); 3. activation/deactivation of primary lighting; 4 . time setting for deactivation of the lights; 5. configuration of light groups and primary lighting, and their touch sensitive zones on the switch.

The microcontroller, mount points, transformer, dimmer, status indicators and touch area were embedded in the switch. The switch prototype was designed on the Arduino platform with the consideration of built-in support for the X10 protocol. The switch includes standard built-in modules: a motion sensor, a light sensor and an infrared sensor (IR), which are optional and can be removed. The modular design of the switch provides the option to extend its functionality with other smart devices and sensors, and by adding modules the switch may be upgraded with additional protocols for integration, Bluetooth, WiFi, ZigBee, INSTEON, and so on. One compromise in the development phase of the switch was the use of a resistive touch-sensitive surface instead of a capacitive one in the prototype version. These panels are much cheaper, are easier to program and can detect touch even when wearing gloves. On the other hand, their multi-touch support is very limited, and they allow less accurate touch control in comparison to capacitive touch panels. However, this is not a major issue in the case of a light switch, as most gestures are single-touch based and interaction with the switch is in most cases crude, and thus touch precision and accuracy are also less important. With the use of a resistive surface we managed to create a prototype that supports almost all the selected gestures. Since our goal was to manage more than six lights and there are a limited number of inputs/outputs on the Arduino UNO board, we decided to use a TLC5940 chip (16 PWM outputs). The modular construction concept of the switch is presented in Fig. 2.

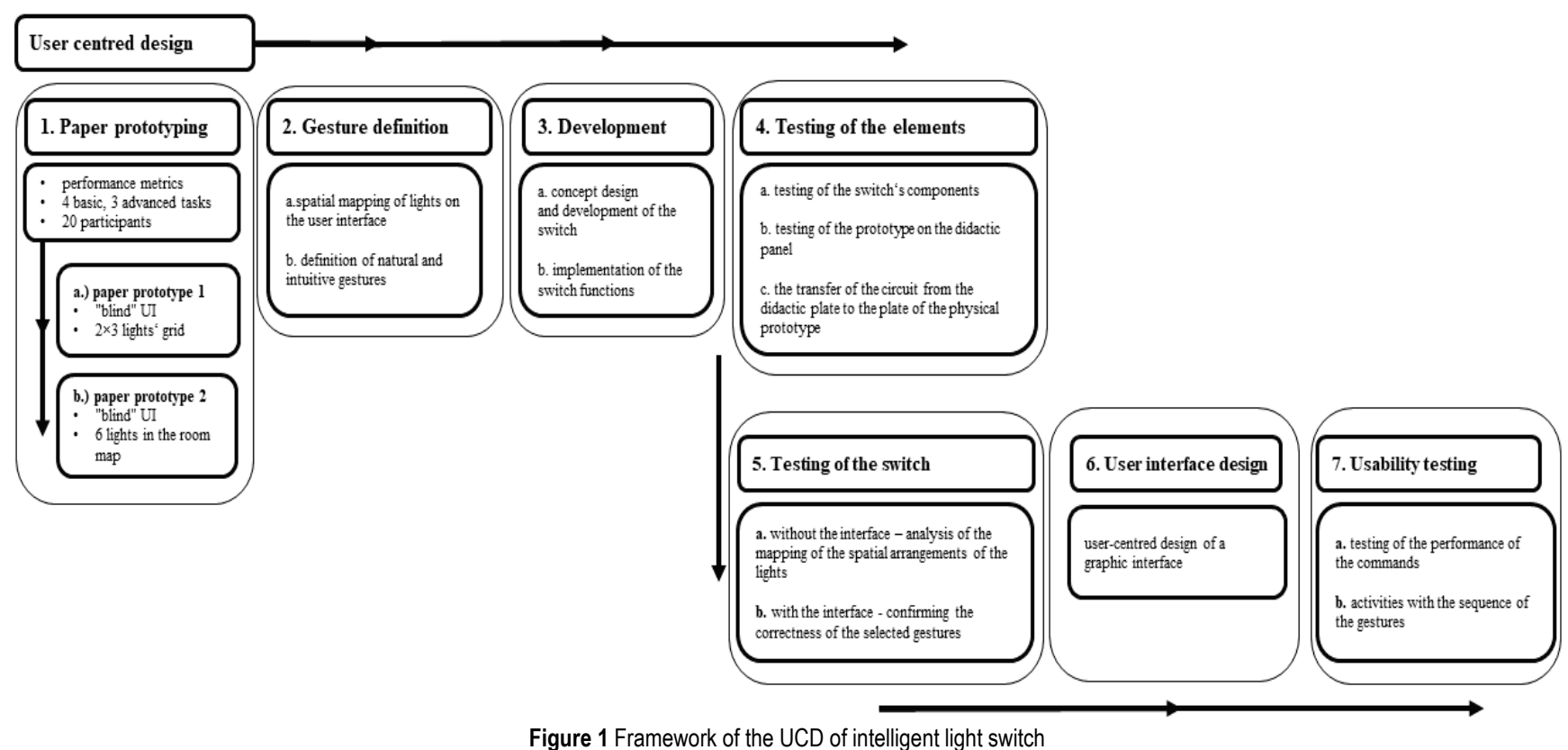

Figure 1 Framework of the UCD of intelligent light switch 
Table 1 Paper prototype and the list of basic (A-D) and advanced (E-G) tasks

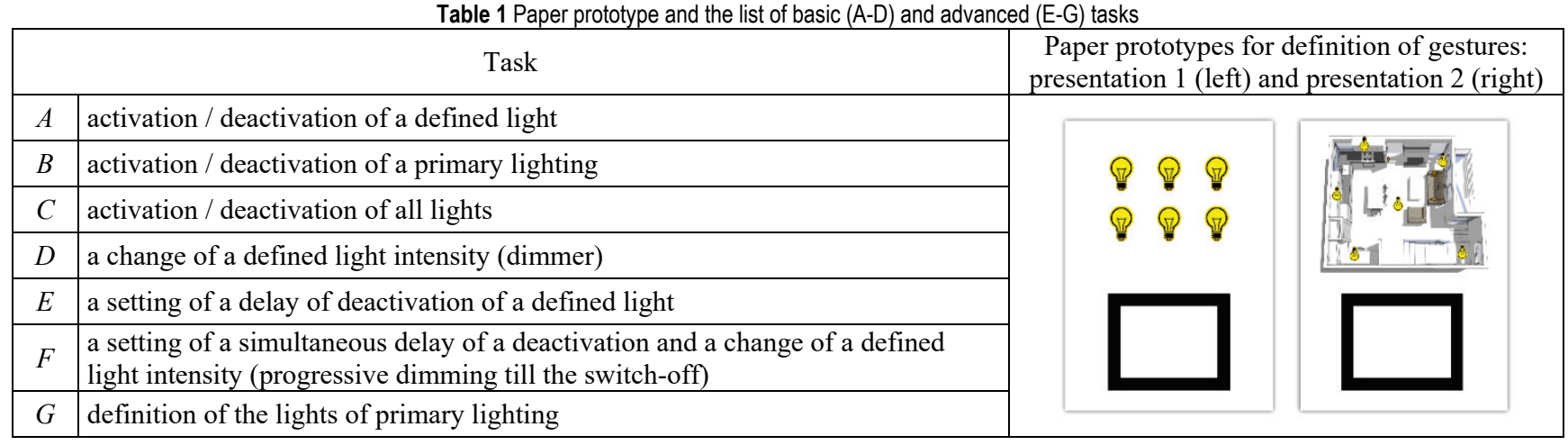

(a)
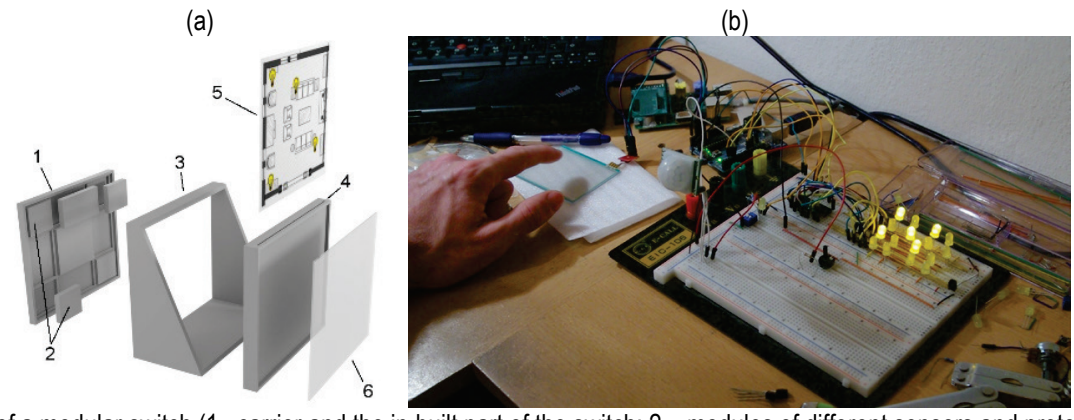

Figure 2(a) Concept design of a modular switch (1- carrier and the in-built part of the switch; 2 - modules of different sensors and protocols; 3 - additional inclined canvas; 4 - slot for the setting of the image; 5 - schematic presentation of the room and light locations; 6 - resistive surface), and b) testing of the prototype on the didactic panel

\subsection{The Prototype on a Didactic Panel}

After successful testing of each part of the switch (touch surface, microcontroller Atmega328, TLC5940 chip, photocell, IR detector, and Velleman K8064 dimmer), all the components were combined and installed on a didactic panel. During testing it was discovered that when using the Arduino UNO platform it was not possible to combine the TLC5940 chip and the IR detector. The library of TLC5940 uses both timer 1 and timer 2, and the IR library also uses timer 2; the Arduino UNO was thus replaced with the development board Teensy2.0++, which has additional timer 3 that was used for integrating the IR detector. Testing of the prototype on the didactic panel is shown in Fig. 2.

The last phase of the development was the transfer of the circuit from the didactic plate to the plate of the physical prototype and the production of the supporting frame. Based on the results of the test with the paper prototypes, the process of UCD continued with the programming of the most commonly suggested gestures as commands. Certain gestures were slightly adapted and divided in two or more separate gestures for one command programmed in the switch in order to increase the usability of the switch and enable the commands to be more universal (Table 5).

\subsection{Usability Testing of the Prototype}

The prototype was first tested as a version without the user interface to discover how quickly the users would be able to learn the gestures needed to operate the lights, and then tested again with the interface to analyse user responses to the switch's usability and the gestures that were defined for the manipulation of the switch's commands.
The usability testing of the prototype was examined using performance metrics obtained from six users [29] in two steps. The first step was the testing of the prototype without the graphical user interface, i.e. without the image of lights below the touch surface of the switch, so that the users' orientation and capabilities with regard to the mapping the spatial arrangement of the lights on the switch were tested. After the analysis of the results of the first step the UCD of a simple graphic interface was performed in Processing, and its usability with regard to controlling the light switch over the network was tested. In both usability testing steps each of the participants was asked to perform basic commands (Tab. 5), and were again video recorded and the results analysed. The participants were also asked to perform three activities (AA-CC) that demanded a longer sequence of gestures: AA. to change the intensity of an individual light, which requires users to perform a sequence of the following gestures: 1.) one-finger tap on a zone where a specific light is defined to turn it on; 2 .) $2 \times$ one-finger taps on a zone where a specific light is defined to select it; 3.) horizontal one-finger slide to change the light intensity; 4.) $2 \times$ one-finger taps on the zone where the light is defined to confirm the change,

BB. to change the intensity of a group of lights (zone), which requires the users to perform a sequence of the following gestures: 1.) one-finger tap on the zone where a specific light is defined to turn it on; 2.) horizontal onefinger slide to change the light intensity; 3.) $2 \times$ one-finger taps on the zone of the light to confirm the change,

CC. to set the delay of a primary illumination for 20 sec, which requires the users to perform a sequence of the following gestures: 1.) vertical/horizontal undefined multifinger slide or $1 \times$ undefined multi-finger tap to activate the primary illumination; 2 .) press and hold ( $>10 \mathrm{~s})$, to enter the settings; 3.) $20 \times$ taps or numerical writing of the number to define the delay in seconds; 4.) press and hold ( $>10$ s), to 
exit the settings; 5.) $2 \times$ one-finger taps to confirm the settings.

\section{RESULTS AND DISCUSSION 3.1 Description of Gestures}

The gestures used in this work were as follows: 1 . gesture $1 \times$ tap means one touch followed by at least $300 \mathrm{~ms}$ without touching. In cases when the next touch occurs within $300 \mathrm{~ms}$, a new touch is added to the total number of touches. For example: if the touchscreen detects one tap that is followed by another after $250 \mathrm{~ms}$, and after this there is no detection of any subsequent taps for $300 \mathrm{~ms}$, the system concludes that the user performed a double tap, $2 \times$ taps; 2. gesture $N$ taps means $N$ individual touches with a spacing greater than $300 \mathrm{~ms}$ that are performed successively; 3 . gesture $N \times$ taps means the performance of several successive touches that were no more than $300 \mathrm{~ms}$ apart. How long the system should wait for the identification of one or more touches is not completely standardised, so in our research the usually implemented definition of time, $300 \mathrm{~ms}$, was applied [30].

\subsection{Results of the Paper Test Prototype}

Tabs. 2 to 4 show the descriptions and number of user suggestions that were given according to tasks $\mathrm{A}-\mathrm{G}$. These tables show the gestures that were used more than once, while those that were suggested by only one user are not given in the text. Some users suggested more than one possible gesture for a task, and so some tasks in the Tables 2 to 4 have more results (No. total) than there were users involved in testing (No. users $=20$ ). Besides, since they were progressing from simple to more difficult tasks, the users sometimes revisited and changed the gestures that they first suggested for the simpler tasks. This was mainly because the users realised that some of the gestures they suggested for the simpler tasks were more suitable for a different purpose, or that they should be used in a different order. Consequently, the results sometimes contain multiple gesture suggestions for the same task from the same user.

Table 2 Results for task A - activation / deactivation of a defined light, and for task B - activation / deactivation of a primary illumination

\begin{tabular}{|l|c|l|l|}
\hline \multicolumn{2}{|l|}{ Task A (No. total = 22) } & Task B (No. total = 24) \\
\hline Gesture & No. & Gesture & No. \\
\hline $1 \times$ one-finger tap in the zone & 15 & $1 \times$ undefined multi-finger tap \\
\hline vertical slide/swipe finger in the zone & 3 & $1 \times$ one-finger tap in the centre \\
\hline $2 \times$ one-finger taps in the zone & 2 & $2 \times$ one-finger taps in the centre \\
\hline \multirow{2}{*}{} & 6 & horizontal slide/swipe finger over the entire area \\
\cline { 2 - 3 } & separate activation of each single light \\
\hline
\end{tabular}

For task A there were three additional gestures that were performed/suggested by only one participant. For task B there were five additional gestures performed/suggested by only one participant.

Table 3 Results for task $C$ - activation and deactivation of all lights and the results for task D - the change in light intensity of a defined light (dimmer)

\begin{tabular}{|l|c|l|l|}
\hline \multicolumn{2}{|l|}{ Task C (No. total = 23) } & No. & Gesture \\
\hline Gesture & 7 & circular gesture with the finger in the zone (o) \\
\hline $1 \times$ undefined multi-finger tap & 2 & horizontal or vertical finger slide out from the zone of the light \\
\hline $1 \times$ long one-finger tap (press and hold) & 2 & $\begin{array}{l}1 \times \text { long one-finger tap (press and hold) for the change of the light intensity } \\
\text { followed by the release of the finger }\end{array}$ \\
\hline $2 \times$ one-finger taps & 2 & $\begin{array}{l}1 \times \text { one-finger tap in the zone for the selection, followed by a vertical finger } \\
\text { slide for the definition of light intensity }\end{array}$ \\
\hline $\begin{array}{l}1 \times \text { one-finger tap for separate activation of each single } \\
\text { light }\end{array}$ & 2 & pinch and zoom in the zone of the light \\
\hline $2 \times$ undefined multi-finger taps & $\begin{array}{l}1 \times \text { one-finger tap in the zone followed by pinch and } \\
\text { zoom for the definition of light intensity }\end{array}$ \\
\hline & $\begin{array}{l}2 \times \text { one-finger taps in the zone for the selection, followed by vertical slide } \\
\text { for the definition of light intensity }\end{array}$ \\
\hline \multirow{2}{*}{2}
\end{tabular}

For task $\mathrm{C}$ there were seven additional gestures that were performed/suggested by only one participant and for task $\mathrm{C}$ there were three additional gestures that were performed/suggested by only one participant.

\subsection{Gestures}

The gestures that were implemented in the switch are presented in Tab. 5 .

\subsection{Results of Usability Testing of the Physical Prototype}

The usability testing of the physical prototype was performed on six users in the final stage of our research. Basic tasks were given to the users and the results revealed that the selection of the programmed gestures was appropriate. Most of the users tried to activate the lights with a single tap, multi-finger taps or with a finger slide over the screen. These gestures were determined for activation of groups of lights and primary illumination, and therefore for all the gestures used the switch reacted adequately to the commands. The most basic gestures were thus easily discoverable, and users quickly learned how to preform basic commands even without the help of a graphical interface. However, the advanced functions were harder for the users to discover or execute, which was expected based on the results of testing the paper prototypes. Furthermore, in further tests it was discovered that the users had properly projected the spatial arrangement of the lights on the switch. When an image of the lights below the touchscreen surface of the switch was added, this became even easier for the users.

Testing of the basic commands showed that the execution of the gestures was $97.7 \%$ accurate when considering the results for all the commands. The commands presented in Tab. 5 were performed correctly (with 100\% success) by all six users in the cases of commands 1-3 and 5-6: 1 (activation/deactivation or light/zone selection), 2 (activation/deactivation of primary 
illumination), 3 (selecting and/or confirmation), 5 (time setting) and 6 (settings enter/exit); and by five users ( 83.3 of success) in the case of command 4 (change of light intensity).

When the users were asked to perform activities that demanded a sequence of gestures, the success rate was $94.4 \%$ overall. In the case of activity AA six users (100\%) executed the sequence of gestures correctly, in the case of activity BB five users $(83.3 \%)$ did so, and in the case of activity $\mathrm{CC}$ all six of the users $(100 \%)$ performed the sequence of gestures correctly.

Some issues were noticed during the testing of the prototype, including the incorrect reaction of the switch to some gestures, despite the users making the appropriate actions. This is probably due to the programming code, which should be made more robust to enable the performance of all the commands regardless of minor deviations in some gestures. For instance, the taps of some users were longer than (the programmed) $300 \mathrm{~ms}$, but short enough to be understood as $1 \times$ one-finger tap from the users' point of view. Additionally, occasional minor disturbances in motion detection caused the selection of the wrong group of lights or the confirmation of a gesture before it had ended.

Physical prototype of the switch and the interactive user interface programmed in Processing are presented Fig. 3. (a)
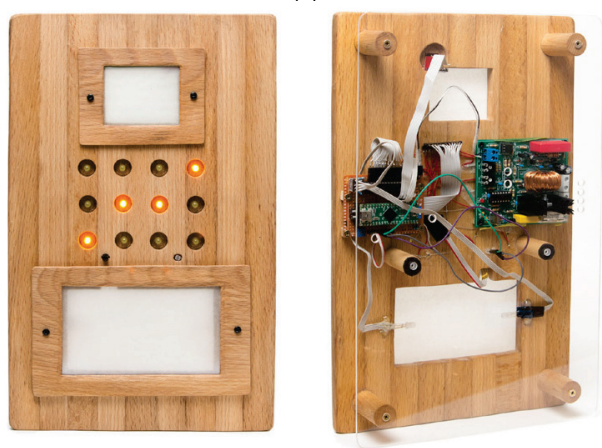

(b)

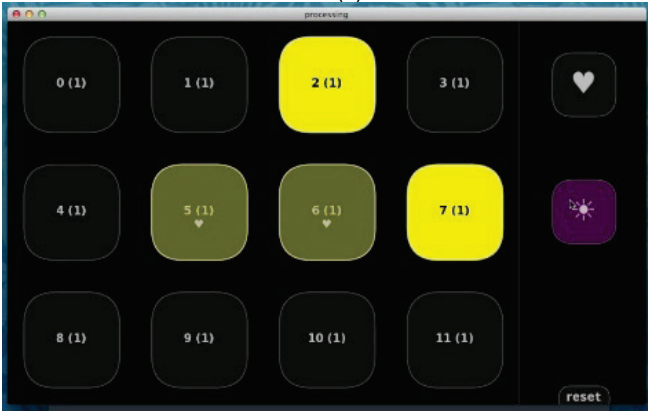

Figure 3(a) Final prototype and (b) a simple interactive user interface programmed in Processing

Table 4 Results for task E - setting of the delay for deactivation of a defined light, results for task F - delay of light deactivation with a change in light intensity, and results for task $G$ - definition of the lights for primary illumination

\begin{tabular}{|c|c|c|c|c|c|}
\hline \multicolumn{2}{|l|}{ Task E (No. total $=20)$} & \multicolumn{2}{|l|}{ Task F (No. total $=\mathbf{2 0})$} & \multicolumn{2}{|l|}{ Task G (No. total $=20)$} \\
\hline Gesture & No. & Gesture & No. & Gesture & No. \\
\hline too difficult, the task was not performed & 5 & $\begin{array}{l}\text { too difficult, the task was not } \\
\text { performed (also task D and E were } \\
\text { too difficult to perform) }\end{array}$ & 9 & too difficult, the task was not performed & 8 \\
\hline $\begin{array}{l}1 \times \text { one-finger tap in the zone for the selection } \\
\text { of the light, followed by writing of the time } \\
\text { delay with the finger }\end{array}$ & 3 & $\begin{array}{l}\text { a combination of the gestures for } \\
\text { tasks D and E }\end{array}$ & 4 & $\begin{array}{l}\text { the use of a menu, windows, and voice } \\
\text { signals (orders) }\end{array}$ & 2 \\
\hline $\begin{array}{l}1 \times \text { long one-finger tap (press and hold) in the } \\
\text { zone, followed by } \mathrm{N} \times \text { taps for definition of the } \\
\text { time delay (interval pre-set) }\end{array}$ & 3 & & & & \\
\hline $\begin{array}{l}2 \times \text { one-finger taps in the zone for the selection, } \\
\text { followed by } \mathrm{N} \times \text { taps to define the time (interval } \\
\text { pre-set) }\end{array}$ & 2 & & & & \\
\hline
\end{tabular}

For task E there were seven additional gestures that were performed/suggested by only one participant, for task $\mathrm{F}$ there were six additional gestures and for task $\mathrm{G}$ there were thirteen additional gestures performed/suggested by only one participant.

\begin{tabular}{|c|c|c|c|c|c|c|c|c|}
\hline \multirow{2}{*}{$\begin{array}{c}\text { Command } \\
\text { Gesture }\end{array}$} & \multirow{2}{*}{$\begin{array}{c}\text { activation/deacti } \\
\text { vation or } \\
\text { light/zone } \\
\text { selection } \\
\\
\text { one-finger tap }\end{array}$} & \multicolumn{2}{|c|}{$\begin{array}{l}\text { activation/deactivation of primary } \\
\text { illumination } \\
\text { (also, deactivation of all lights) }\end{array}$} & \multirow{2}{*}{$\begin{array}{c}\begin{array}{c}\text { selection } \\
\text { and/or } \\
\text { confirmation }\end{array} \\
\begin{array}{c}2 \times \text { one-finger } \\
\text { taps }\end{array}\end{array}$} & \multirow{2}{*}{$\begin{array}{c}\begin{array}{c}\text { change of light } \\
\text { intensity }\end{array} \\
\begin{array}{c}\text { horizontal } \\
\text { one-finger } \\
\text { slide }\end{array}\end{array}$} & \multicolumn{2}{|c|}{ setting time } & \multirow{2}{*}{$\begin{array}{c}\begin{array}{c}\text { enter/exit } \\
\text { settings }\end{array} \\
\begin{array}{c}\text { press and holc } \\
(>10 \mathrm{~s})\end{array}\end{array}$} \\
\hline & & $\begin{array}{l}\text { vertical/horizontal } \\
\text { undefined multi- } \\
\text { finger slide }\end{array}$ & $\begin{array}{c}1 \times \\
\text { undefined } \\
\text { multi-finger } \\
\text { tap }\end{array}$ & & & $\begin{array}{l}\mathrm{N} \times \text { taps (add a } \\
\text { pre-set time } \\
\text { interval for } \\
\text { each tap) }\end{array}$ & $\begin{array}{c}\text { numerical } \\
\text { writing of the } \\
\text { number for time } \\
\text { definition }\end{array}$ & \\
\hline $\begin{array}{c}\text { Graphical } \\
\text { present. } \\
{[31]}\end{array}$ & & & & & & & & \\
\hline
\end{tabular}

\section{CONCLUSIONS}

In this research it was demonstrated that UCD is a successful method for the gesture definition, development and production of an expandable touch-based light switch. UCD was also used as a workflow concept in our experimental procedure that enabled the production of a smart switch with satisfactory usability and ease of use, as well as an intuitive user interface. Moreover, the system was flexible and open enough to enable the further updates and improvement of its stability and intelligence. The smart light switch is also suitable for use in existing residential environments.

In the experimental part it was found that the paper prototype is a valuable methodology in user-centred design and that the gestures with some minor simplifications and 
modifications (such as the grouping of similar suggestions) could be installed on the smart device. The analysis and testing of the users' habits, along with their suggestions for interactions before the production and implementation of the results in the switch prototype, enabled the definition of appropriate intuitive gestures and development of the concept design and implementation of the switch functions. Usability testing of the switch proved that the user-interface design enabled effective use of the product and increased the success of user interactions with the switch. It should be noted here that two further improvements would upgrade the prototype: enhancing both the robustness of the programming code regarding the deviations in some gestures, and the sensitivity of the touch surface that caused occasional errors in the selection of a group of lights. However, we can still conclude that, with a fully working prototype as the result, this study proved that it is possible to make a touch-based light switch, without a screen, and one that is easy to use and can be integrated into both non-intelligent or intelligent home environments.

\section{REFERENCES}

[1] Granzer, W. (2010). Secure Communication in Home and Building Automation Systems. Vienna : University of Technology, Institute of Computer Aided Automation, Automation Systems Group, 1-4.

[2] Smart home: definition of smart home. Oxford dictionary, British \& World English.

http://www.oxforddictionaries.com/definition/english/smart -home. (15.9.2018)

[3] Umberger, M. (2005). Načrtovanje inteligentnega sistema za upravljanje energetsko varčne hiše. Diploma thesis. Ljubljana, p. 8. (in Slovenian)

[4] Cisco Visualization | The Internet of Things. Cisco. http://share.cisco.com/internet-ofthings.html. (15.9.2018)

[5] Protocol Standardization, aka Internet of Things Red Herring. 2015. http://postscapes.com/iot-voices/insights/ protocol-standardization-aka-internet-ofthings-red-herring/. (15.9.2018)

[6] X10 Basics. X10. 2016. http://www.x10.com/x10basics.html. (15.9.2018)

[7] Universal powerline bus. Build Your Smart Home.2016. 2015. http://buildyoursmarthome.co/home-automation/ protocols/universal-powerline-bus/ (10.8.2017)

[8] KNX (standard). (2016). http://www.knx.org/knx-en/knx/ association/what-is-knx/ (15.9.2018)

[9] Introduction to the LONWORKS ${ }^{\circledR}$ System. Echelon Corporation. 1999. http://www.echelon.com/support/ documentation/manuals/general/078-0183-01A.pdf (18.8.2018)

[10] INSTEO Details Whitepaper. INSTEON. (2016). http://cache.insteon.com/pdf/INSTEONCompared.pdf

[11] ZigBee. ZigBee Alliance. (2015). http://www.zigbee.org/ what-is-zigbee/ (18.8.2018)

[12] Z-Wave. V Z-Wave. 2016. http://www.Z-wave.com/ (18.8.2018)

[13] Jia, L., Afshari, S., Mishera, S., \& Radke, R. J. (2014). Simulation for pre-visualizing and tuning lighting controller behavior. Energy Build, 70(2), 287-302. https://doi.org/10.1016/j.enbuild.2013.11.063

[14] In living color: Ars reviews the hacker-approved Philips Hue LEDs. ArsTechnica. (2012). http://arstechnica.com/gadgets/ 2012/11/in-living-color-ars-reviews-the-hackerapprovedphilips-hue-leds/ (10.8.2017)
[15] How hue works - Philips hue API. Philips Hue. (2016). http://developers.meethue.com/howhueworks.html (12.8.2017)

[16] LIFX - The lightbulb reinvented. LIFX. (2016). http://lifx.co/ (18.8.2018)

[17] iLumi. (2016). http://ilumi.co/ (18.8.2018)

[18] Wireless Lighting Control, Wi-Fi Light Dimmer. Plum. (2017). http://plumlife.com/\#lightpad (18.8.2018)

[19] Rath Kumar, D. (2016). Arduino Based: Smart Light Control System. International Journal of Engineering Research and General Sciences, 4(2), 748-790.

[20] Barghi, A., Kosari Reza, A., \& Shokri, M. (2014). Intelligent lighting control with LEDS for smart home. Proceedings of the Smart Grid Conference (SGC), Teheran, Iran. https://doi.org/10.1109/SGC.2014.7090861

[21] Mowad, E. L, Abd, M., Fathy, A., \& Hafez, A. (2014). Smart Home Automated Control System Using Android Application and Microcontroller. International Journal of Scientific \& Engineering Research, 5(5), 935-939.

[22] Wen-Tsai, S. \& Jia-Syun, L. (2013). Design and Implementation of a smart LED Lightning System Using a Self-Adaptive Weighted Data Fusion Algorithm. Sensors, 13, 16915-16393. https://doi.org/10.3390/s131216915

[23] Parkash, V. P. \& Dandu, R. (2016). Internet of Things Based Intelligent Street Lighting System for Smart City. International Journal of Innovative Research in Sciences, Engineering and Technology, 5(5), 7684-7691.

[24] Zanella, A., Bui, N., \& Castellani, A. (2014). Internet of Things for Smart Cities. IEEE Internet of Things Journal, 1(1), 22-32. https://doi.org/10.1109/JIOT.2014.2306328

[25] ISO 9241-210:2010 Ergonomics of human-system interaction - part 210: Human-centred design for interactive systems.

[26] Nielsen, J. (2014). Usability 101: introduction to usability. http://www.nngroup.com/articles/usability-101-introduction -to-usability/ (20.9.2018)

[27] Albert, W. \& Tullis, T. (2013). Measuring the User Experience: Collecting, Analyzing, and Presenting Usability Metrics, $2^{\text {nd }}$ Ed., Morgan Kaufmann.

[28] Dillon, A. (2014). User interface design. MacMillan Encyclopedia of Cognitive Science. https://www.ischool.utexas.edu/ adillon/BookChapters/Use r\%20Interface\%20Design_files/User\%20Interface\%20Desi gn.htm (20.9.2018)

[29] Nielsen, J. (2000). Why You Only Need to Test with 5 Users, https://www.nngroup.com/articles/why-you-only-need-totest-with-5-users/ (12.10.2018)

[30] Web. Updates. (2013). https://developers.google.com/web/ updates/2013/12/300ms-tap-delay-gone-away (20.9.2018)

[31] UX, User Experience. (2016). https://ux.stackexchange.com /questions/76972/research-on-the-most-intuitive-gesturesfor-mobile-devices (20.9.2018)

\section{Contact information:}

\section{Boštjan SENIČAR, MSc}

University of Ljubljana, Faculty of Natural Sciences and Engineering,

Aškerčeva 012, 1000 Ljubljana, Slovenia

bostjan.senicar@gmail.com

\section{Helena GABRIJELČIČ TOMC, PhD}

Corresponding author

University of Ljubljana, Faculty of Natural Sciences and Engineering, Aškerčeva 012, 1000 Ljubljana, Slovenia

helena.gabrijelcic@ntf.uni-lj.si 